Opinion

\title{
The Medical Community Should Make the Unified Disease Concept, Definition, and Diagnostic Criterion of Medically Un- explained Pain (or Symptoms)
}

Katsuhiro Toda ${ }^{*}$

How to cite this paper: Toda, $\mathrm{K}$. (2021). The Medical Community Should Make the Unified Disease Concept, Definition, and Diagnostic Criterion of Medically Unexplained Pain (or Symptoms). Open Journal of Medical Sciences, 1(1), 43-44. Retrieved from https://www.scipublications.com/journal/index.php/ojms/article/view/145

Received: September 18, 2021 Accepted: October 19, 2021 Published: October 19, 2021

Copyright: (c) 2021 by the authors. Submitted for possible open access publication under the terms and conditions of the Creative Commons Attribution (CC BY) license (http://creativecommons.org/licenses /by/4.0/).
Department of Orthopedic Surgery, Hiroshima Clinic. 4th floor Fuji Ground, 2-1, Takara-machi, Naka-ku, Hiroshima, 730-0044, Japan

${ }^{*}$ Correspondence: goutattack@yahoo.co.jp

\begin{abstract}
MUP (medically unexplained pain) is a pain whose cause cannot be determined by diagnostic imaging, blood test, physical examination and past medical history, etc. Many departments have independently created the concept, definition, and diagnostic criterion in MUP. This leads to an abnormal situation in which each department has different disease concepts and diagnostic criteria. It is out-of-the-ordinary that physicians from the different departments make different diagnoses in the same patient. MUP has caused confusion in clinical practice. The medical community should make the unified disease concept, definition, and diagnostic criterion of MUP. For this purpose, the various scientific organizations involved in MUP need to discuss. In this case, the first priority should not be the majority vote, but the treatment outcomes. The solution to a medical controversy is to choose a medical theory or treatment method that produces better long-term results.
\end{abstract}

Keywords: Medically Unexplained Pain; Medically Unexplained Symptom; Fibromyalgia; Chronic Widespread Pain; Chronic Regional Pain; Somatoform Disorder; Somatic Symptom Disorder; The World Psychiatric Association; The American Psychiatric Association; The International Association For The Study of Pain

The terms medically unexplained pain (MUP) and medically unexplained symptoms (MUS) have caused confusion in the medical community. MUS is a combination of MUP and medically unexplained non-pain symptoms. The term MUP is used in this article for simplicity.

MUP is a pain whose cause cannot be determined by diagnostic imaging, blood test, physical examination and past medical history, etc. It is out-of-the-ordinary that physicians from the different departments make different diagnoses in the same patient. Pain due to fracture, compression of the nerve, and local infection is medically explained pain. MUP has caused confusion in clinical practice. Because all clinical departments participated in determining the concept and diagnostic criterion of cancer, there is little confusion at least in diagnosis. Many departments have independently created the concept, definition, and diagnostic criterion in MUP. This leads to an abnormal situation in which each department has different disease concepts and diagnostic criteria. It is out-of-theordinary that physicians from the different departments make different diagnoses in the same patient.

Many departments of the medical community have made their own disease concepts and diagnostic criteria for MUP. Department of psychiatry defined somatoform disorder (SD) or somatic symptom disorder (SDD). Psychogenic pain and masked depression are concepts similar to SD or SDD. SD is a diagnosis based on the diagnostic and statistical manual of mental disorders 4 th edition text revision (DSM-IV-TR). SDD is a diagnosis 
based on the new diagnostic and statistical manual of mental disorders, Fifth Edition (DSM-5). Department of chronic pain defined fibromyalgia (FM) and incomplete form of FM (I-FM) (chronic widespread pain (CWP) and chronic regional pain (CRP)). Some physicians defined myofascial pain syndrome (MPS). Chronic pelvic pain syndrome, chronic vulvar pain syndrome, idiopathic orofacial pain, atypical odontalgia, atypical facial pain, burning mouth syndrome, and tension-type headache are MUP of the localized region. Some symptoms of autonomic dysregulation, menopause disorder, and dysmenorrhea are MUP.

Some physicians are unaware of the confusion regarding MUP. Several medical articles and medical books have discussed the distinction between FM and SD. If we know the confusion about MUP, differentiating between FM and SD is worthless and a waste of time.

The medical community should unify the disease concept, definition, and diagnostic criterion of MUP. For this purpose, the various scientific organizations involved in MUP need to discuss. In this case, the first priority should not be the majority vote, but the treatment outcomes. The solution to a medical controversy is to choose a medical theory or treatment method that produces better long-term results.

The first priority of the unification is the unification of FM (or I-FM) and SD (or SDD). Some studies showed that FM and SD may be the same disease (disorder) [1,2]. The World Psychiatric Association (WPA) is an international scientific organization of the department of psychiatry. The International Association for the Study of Pain (IASP) is an international scientific organization of the department of pain. I submitted a medical paper to the American Psychiatric Association (APA) requesting that the APA and IASP discuss the disease concept of MUP, however, it was rejected. I hope that the WPA (or APA) and IASP discuss the disease concept of MUP.

I believe that central sensitivity syndrome (CSS) is the core of MUP. The prevalence of FM is approximately $2 \%$ [3]. The prevalence of CWP including FM is approximately $10 \%$ [4]. The prevalence of CRP is 1-2 times as frequent as that of CWP [5]. In other words, the prevalence of FM including I-FM is at least $20 \%$. The treatment of I-FM is identical to the treatment of FM. I believe that FM, including I-FM, is the core of CSS in terms of the number of effective treatments, a variety of symptoms, prevalence, and treatment outcomes.

In conclusions, many departments have independently created the concept, definition, and diagnostic criterion in MUP. Physicians from the different departments make different diagnoses in the same patient. MUP has caused confusion in clinical practice. The medical community should make the unified disease concept, definition, and diagnostic criterion of MUP. For this purpose, the various scientific organizations involved in MUP need to discuss.

Funding: No funding.

Conflicts of interest: My daughter is an employee of Nippon Zoki Pharmaceutical Co., Ltd.

Ethics approval: Not applicable.

\section{References}

[1] Atmaca, M. Neuro-imaging in Somatoform Disorders: A review. Turk. Psikiyatri. Derg. 2012, 23, $274-280$.

[2] Hauser, W.; Burgmer, M.; Kollner, V.; Schaefert, R.; Eich, W.; Hausteiner-Wiehle. C,; Henningsen, P. Fibromyalgia syndrome as a psychosomatic disorder - Diagnosis and therapy according to current evidence-based guidelines. Z. Psychosom. Med. Psychother. 2013, 59, 132-152.

[3] Heidari, F.; Afshari, M.; Moosazadeh, M. Prevalence of fibromyalgia in general population and patients, a systematic review and meta-analysis. Rheumatol. Int. 2017, 37, 1527-1539.

[4] Mansfield, K.E.; Sim, J.; Jordan, J.L.; Jordan, K.P. A systematic review and meta-analysis of the prevalence of chronic widespread pain in the general population. Pain. 2016, 157, 55-64.

[5] Toda, K.; Harada, T. Prevalence, classification, and etiology of pain in Parkinson's disease: association between Parkinson's disease and fibromyalgia or chronic widespread pain. Tohoku. J. Exp. Med. 2010, 222, 1-5. 